\title{
Effect of the Gas Price Surges on User Activity in the DAOs of the Ethereum Blockchain
}

\author{
Faqir-Rhazoui, Youssef \\ yelfaqir@ucm.es \\ Departamento de Ing. del Software e Inteligencia Artificial. \\ Universidad Complutense de Madrid \\ Madrid, Spain \\ Arroyo, Javier \\ javier.arroyo@fdi.ucm.es \\ Institute of Knowledge Technology, Universidad \\ Complutense de Madrid \\ Madrid, Spain
}

\begin{abstract}
Blockchain technology has enabled a thriving emergent ecosystem of tools and communities actively using decentralized systems. However, most blockchain infrastructure (e.g. Ethereum) requires users to pay some fees to execute their desired actions in these novel online services. To which extent an increase in the price of such fees negatively affects user activity? Would significant price surges deter users from using blockchain-enabled online services? In this work, we study the 2020 surge of transaction fee price in the Ethereum network, and analyze how that affected user activities. Our use cases are the blockchain-enabled Decentralized Autonomous Organizations (DAOs) from the platforms DAOstack and DAOhaus. Thus, we analyzed 5,580 transactions from 7,825 users grouped in 191 DAO communities, using a VAR model with a daily time series of the average fee value and the DAO operations. Our results show just a minor influence of the fee (gas) price and the activity of DAO users. The insensitivity of the activity to the fee price is an anomaly in a supposedly self-regulated market, and we consider this should be tackled in future implementations.
\end{abstract}

\section{CCS CONCEPTS}

- Human-centered computing $\rightarrow$ Empirical studies in collaborative and social computing; Collaborative and social computing design and evaluation methods.

\section{KEYWORDS}

Blockchain, Ethereum, Decentralized Autonomous Organizations, DAOs, Causality, Gas Price, DeFi

Permission to make digital or hard copies of all or part of this work for personal or classroom use is granted without fee provided that copies are not made or distributed for profit or commercial advantage and that copies bear this notice and the full citation on the first page. Copyrights for components of this work owned by others than the author(s) must be honored. Abstracting with credit is permitted. To copy otherwise, or republish, to post on servers or to redistribute to lists, requires prior specific permission and/or a fee. Request permissions from permissions@acm.org.

CHI '21 Extended Abstracts, May 8-13, 2021, Yokohama, Japan

(C) 2021 Copyright held by the owner/author(s). Publication rights licensed to ACM ACM ISBN 978-1-4503-8095-9/21/05 . \$ \$15.00

https://doi.org/10.1145/3411763.3451755

\author{
Ariza-Garzón, Miller-Janny \\ millerar@ucm.es
}

Departamento de Ing. del Software e Inteligencia Artificial.

Universidad Complutense de Madrid

Madrid, Spain

\author{
Hassan, Samer \\ shassan@cyber.harvard.edu \\ Berkman Klein Center, Harvard University \\ Cambridge, Massachusetts, USA \\ Institute of Knowledge Technology, Universidad \\ Complutense de Madrid \\ Madrid, Spain
}

\section{ACM Reference Format:}

Faqir-Rhazoui, Youssef, Ariza-Garzón, Miller-Janny, Arroyo, Javier, and Hassan, Samer. 2021. Effect of the Gas Price Surges on User Activity in the DAOs of the Ethereum Blockchain. In CHI Conference on Human Factors in Computing Systems Extended Abstracts (CHI '21 Extended Abstracts), May 8-13, 2021, Yokohama, Japan. ACM, New York, NY, USA, 7 pages. https://doi.org/10.1145/3411763.3451755

\section{INTRODUCTION}

Since the advent of Bitcoin in 2008 [22], the technology that powers it, blockchain, has been growing in popularity and adoption. This technology enables new forms of online services which rely on a decentralized infrastructure. Some of the properties of blockchain networks include: transparency of the operations performed; immutability of the history of transactions; and resilience since the chain of blocks that contain the operation data is ensured through cryptographic means $[13,28]$.

Beyond cryptocurrencies, the most widely used blockchain project is the Ethereum computing platform, which enables developers to build distributed apps on top of its general-purpose blockchain. Ethereum enables the execution of self-enforced and automated general-purpose code, "smart contracts", without relying on a thirdparty to host and control the online service [12]. Based on these smart contracts, blockchain enables new forms of decentralized governance $[10,17]$. In fact, a complex set of smart contracts may be set up in such a way as to make it possible for multiple parties to interact with each other, forming a Decentralized Autonomous Organization (DAO).

A DAO is a blockchain-based system that enables people to coordinate and self-govern themselves mediated by a set of selfexecuting rules deployed on a public blockchain, and whose governance is decentralized (i.e. independent from central control) [15]. DAOs may hold cryptocurrency and other crypto-assets, and therefore can buy/sell resources, or pay "employees" [4]. It is common for DAO members to reach community consensus (e.g. about where to allocate money) through some sort of voting mechanism, which is typically one of the main DAO interactions in a blockchain 
$[5,11] .{ }^{1}$ However, the final voting is usually performed in the DAO blockchain, enabling it to be public and transparent.

Any interaction in the blockchain, being a cryptocurrency transaction, a voting, or any change to the state of the blockchain program, implies some computation to be performed by the Ethereum network. As opposed to traditional centralized web hosts, such computation is not performed by a central server, but by the Ethereum distributed network of nodes. This implies there is no monthly payment for hosting like in traditional web services. However, it also implies someone else should compensate those nodes for their work (computation), which is usually the user. That is, interacting with Ethereum apps often implies a micro-payment (fees) from the user triggering the operation[4]. This is a novel environment, in which participation, e.g. voting, implies a monetary cost for the user. This is an unusual human-computer interaction setting that presents the immediate research question: is such monetary cost an obstacle for users to keep interacting with blockchain-enabled online services? Or, for current users, would a sensible increase in the price of operation deter them from further interactions?

In order to explore that, we need to delve more into how Ethereum fees work. Transaction ${ }^{2}$ fees vary depending on the amount of code to be executed, and its complexity [30]. In order to measure the computational effort required, Ethereum uses the unit "gas". The price of gas fluctuates depending on the activity of the network and the work of the nodes performing the computation ("miners"). In practice, this works following market dynamics, in which a peak of demand of computation in the network implies many operations competing to be computed first... which raises the cost of the gas price (the fees), i.e. the amount to be paid for your transaction to go through before others. Eventually, the system is implemented with the expectation of self-regulation, i.e. that high fees attract more miners to offer computation services, adapting to the growing demand and stabilizing the gas price.

Thus, we hypothesize that increases of the gas price would impact negatively the amount of operations performed by users. Particularly, we want to ascertain whether such increases affect to governance operations in DAOs, not including other Ethereum operations, especially, investments where the expected returns could compensate the fee increase.

These issues have been under-studied in the current literature. There are studies on the factors that affect price and transaction fees in both Ethereum and Bitcoin, rather than how the price affects user interactions. For instance, a study that explain the price movements of Bitcoin as an investment asset and its qualities of money as a means of payment [24]. Likewise, there is Granger causality between the total amount of miners in the Ethereum network and the final gas price [23]. Similarly, the volume of transaction fees in Bitcoin is driven more by social conventions formed by some actors rather than the market protocol [20]. Regarding the transaction period time, it is not directly related to the fee value [7], even if another study found that, according to a game-theoretic model, waiting times were a significant factor [8]. Again, these results do

\footnotetext{
${ }^{1}$ Users make use of other channels to interact and discuss issues, such as forums or chat groups. However, those are not registered in the blockchain.

${ }^{2}$ Operations are often named "transactions" in this context, inherited from the original uses of blockchain as a cryptocurrency ledger.
}

not cover the scope of our work, which explores the effect on user interactions.

\section{THE CURRENT CONTEXT}

Figure 1 shows that the evolution of gas price during the second half of 2020 has been increasing in an unprecedented manner. For example, in September 2019 the average price was $0.0225 \mathrm{ETH}$ ( $\$ 4.8$ at the time), and one year later it was 0.193ETH ( $\$ 74.9$ at the time), that is, a $8500 \%$ increase. ${ }^{3}$ And on top of that, the price was highly volatile: the standard deviation in September 2020 was $2500 \%$ increase that in September 2019 (0.1216ETH and 0.00474ETH, respectively).

The main cause of this extreme behavior is network congestion, due to an increase of demand generated by the booming of Decentralized Finance (DeFi) applications, especially from March 2020 $[1,16]$. Most current DeFi services run on Ethereum, and thus share a blockchain, a pool of miners, and demand from the network, with all other Ethereum apps. DeFi is driven by investors which expect high returns of capital, and therefore are willing to pay much higher gas prices for their transactions to go through. For those not aiming to invest but to operate in decentralized applications, this may be burdensome, and it has started triggering the bankruptcy of some services [29]. Thus, DAOs constitute an appropriate use case to analyze how non-investment operations in Ethereum are affected by surges in gas prices. In particular, how gas price surge affects DAO activity?

The platforms that facilitate the deployment of DAOs and that are responsible of the growth of the DAO phenomenom are Aragon, DAOstack and DAOhaus [9]. As we will explain below, we focus our study on the last two platforms.

\section{GAS: THE ETHEREUM TRANSACTION FEE}

Transaction costs are usually not expressed in Ether (ETH) but in smaller units named wei and Gwei, where $1 E T H=10^{1} 8 w e i$, and $1 E T H=10^{9}$ Gwei. As mentioned above, the gas price varies according to the demand of computational resources in the Ethereum network, and thus with the number of transactions to process in a given time. We will explain it further. The block-chain is essentially a chain of blocks, where each block compiles a certain number of transactions. Miners are responsible for processing new transactions, compiling them in a new block, which is then added to the chain. The miners collect the fees linked to each transaction of their block, and will typically process first those transactions with higher fees (competing with other miners to process them). The number of transactions that can be inserted in a block is tied to the total number of gas that those transactions spend. Each block has a limit of gas which transactions can use, and this limit is set by the miners each block [31]. Increasing the block gas limit will also take more time to propagate the changes around the network. If those blocks take more time to be processed, it will also take more time to discover new blocks, but it also will reduce the fee's value. However, it is essential to keep the block gas limit as low as reasonably possible in order to maximize the network decentralization [1].

\footnotetext{
${ }^{3}$ The Ether price (ETH), Ethereum's cryptocurrency, has also grown in this time. Still, the gas price has increased independently from Ether's growth. The equivalency to dollars is performed taking into account the price of Ether at that given time, seeing an increase of the cost in dollars for the same operation $1500 \%$.
} 


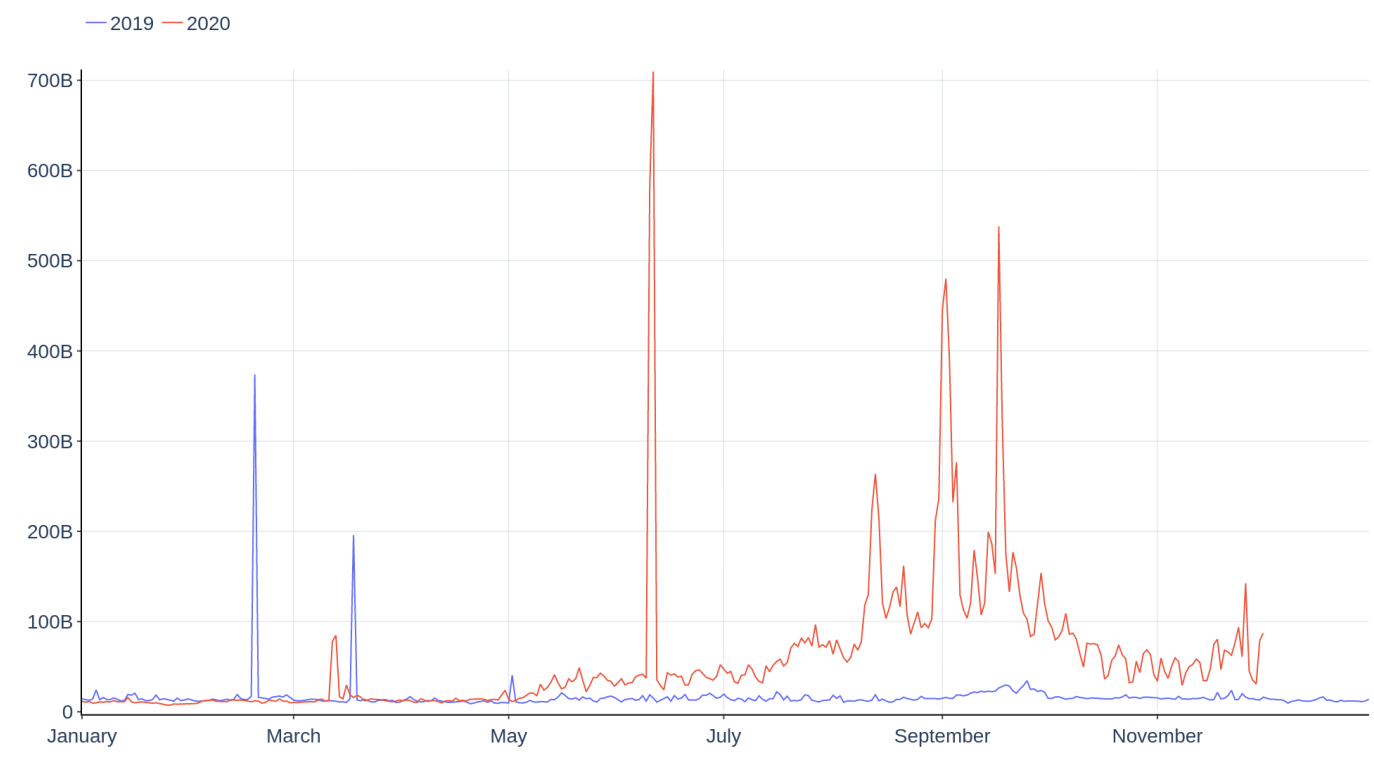

Figure 1: Average gas price in Wei along 2019 (in blue) and 2020 (in red) until November.

Since there is no simple way to know how much gas fee a user will eventually have to pay for a transaction (since fees are dynamic), users can set a gas limit to pay. However, a low limit may lead to a long time to accomplish the transaction, or even worse, the transaction may not execute, and the user will lose the fees spent in the attempt. On the contrary, a high gas limit may derive in an overpriced fee. Due to that, there are many tools ${ }^{4}$ that usually provide an estimated gas price depending on the speed demanded, and the cost of the code to be executed [23].

Finally, Ether can be bought with fiat money at a variable exchange rate. As of 5th January 20211 ETH $=1097.69$ USD. However, our analysis focuses on the Ether cost without considering the USD-cryptocurrency exchange fluctuations.

\section{DATA AND METHODS}

\subsection{Data description}

Our analysis considers the period from May 2020 to December 2020 where both the level and the volatility of the Ethereum gas price increased significantly, which could affect the activity in DAOs, according to our research hypothesis.

We retrieved the daily average gas prices from Etherscan. ${ }^{5}$ Since the gas price time series had trend and volatility, we transformed it to logarithmic returns, as it is usually done with price time series in Finance. Thus, we dealt with the logarithmic returns time series that is easier for model fitting and also has a clear economic interpretation.The daily log-returns of the gas price, i.e., ret_gas $t_{t}=\ln \left(\right.$ gas_price $_{t} /$ gas_price $\left._{t-1}\right)$, incorporate information on the growth and decrease movements of prices that is roughly similar to percentage increases and decreases.

\footnotetext{
${ }^{4}$ ETH Gas Station: https://ethgasstation.info/, or Etherchain: https://www.etherchain. org/

${ }^{5}$ https://etherscan.io/
}

For measuring the activity of a DAO ecosystem, we considered the daily ratio of activity per registered user, that is, the quotient between the number of actions performed in a DAO ecosystem during a given day and the number of members registered in the DAO ecosystem until that day. We analyzed the activity as a ratio, because the number of DAO members increased significantly in one of the ecosystems considered during the period studied. We wanted to remove the impact of the member growth from our analysis.

We analyse two DAO ecosystems, DAOstack and DAOhaus, because their APIs provide all the data needed. ${ }^{6}$ They sum 5,580 transactions, 7,825 users, and 191 DAOs in the period analyzed. However, we could not include Aragon, the most popular platform, because its API does not provide the user registration date needed to compute a ratio.

Regarding the measurement of activity in a DAO, we use the definition in [9], where for DAOstack they consider the following actions: create a proposal, vote for a proposal and stake for a proposal. Similarly for DAOhaus we considered the following actions: create a proposal, vote for a proposal and leave the DAO (known as rage-quitting).

\subsection{Methods}

We analyzed the dependence between gas price and DAO activity using econometric methods. The original time series have weekly seasonality, that is the presence of regular weekly variations. Since seasonality can bias the results of dependence analysis, we performed a seasonal decomposition of the time series and removed the seasonal component.

Before the model estimation, we evaluated the stationarity of the de-seasonalized time series, that is, that the mean and variance

${ }^{6}$ DAOstack: https://thegraph.com/explorer/subgraph/daostack/master, DAOhaus: https://thegraph.com/explorer/subgraph/odyssy-automaton/daohaus 
and the autocorrelation structure of the series do not change over time. If the time series are stationary we can interpret the results of the VAR models with more certainty. We use two unit-root tests: Augmented Dickey-Fuller (ADF) and a non-parametric alternative, Phillips-Perron (PP) test, and their critical values are derived from $[18,19]$.

For the dependence analysis, we used the VAR models [26]. In a VAR model of order $p$, each variable is modeled as a linear combination of past values of itself and the past values of the other variables considered. $\operatorname{Our} \operatorname{VAR}(p)$ is:

$$
y_{t}=v+A_{1} y_{t-1}+\ldots+A_{p} y_{t-p}+u_{t}
$$

where the vector of variables is $y_{t}=\left(\text { ret_gas }_{t} \text {, activity }\right)^{\prime}$, the (2x2) matrices of parameters are $v, A_{1}, \ldots, A_{p}$, and the error process $u_{t}=\left(u_{1 t}, u_{2 t}\right)^{\prime}$ is 2-dimensional white noise, with covariance matrix $E\left(u_{t} u_{t}^{\prime}\right)=\sum_{u}$, that is $u_{t} \sim\left(0, \sum_{u}\right)$.

VAR models represent the dependence patterns between the set of variables considered and offer the possibility of evaluating the statistical "causality" that can exist between them. For that purpose, we used three econometric tools: orthogonalized impulse response functions, the decomposition of the variance of the forecast error and Granger's causality test under the fitted model structure.

The plots of the orthogonalized impulse response functions represent the dynamics generated in the system variables, In our case, we are interested in the activity, in the face of the impact of a random shock on the price of gas. We will quantify these shocks' effects, the sign of them, and evaluate whether they are transient or permanent.

The decomposition of the variance determines how the forecast error in each variable can be attributed to its innovations or innovations of other variables. It will help us to assess the sensitivity of the activity rate to price changes.

The impulse response functions and the decomposition of the variance are used to assess the existence, sign and direction of the relationship of the variables. We complement such statistical tools with the Granger-causality test [14] to reassess the relationship's presence with a further method. In particular, whether the gas price changes cause activity movements in Granger's sense, which is predictive causality.

We estimated a $\operatorname{VAR}(p)$ for each DAO ecosystem, choosing $p$ by optimizing the Akaike information criterion (AIC) and Bayesian information criterion (BIC). We selected a $p$ order that minimizes such criteria evaluated over a range of orders. We assessed the model by checking that the residues behave as multivariate white noise. For the latter, we used the autocorrelation function graphs and a portmanteu test for checking the whiteness of the residuals for up to 20 lags.

More details on these methods can be found in [2]. For the statistical analysis, we used the statsmodels package [27]. The data, the code used to retrieve it and the statistical analysis code are publicly available under open licenses. ${ }^{7}$

\section{RESULTS}

Regarding the stationarity of the time series, Table 1 shows that the gas return series is stationary according to the tests considered at

\footnotetext{
${ }^{7}$ GitHub repository: https://github.com/Grasia/gas-dao-activity
}

all significance levels. Regarding the two activity rate time series, they are stationary according to the ADF and PP tests (significant at $1 \%$ and $5 \%$, respectively). Thus, we have the necessary stationary conditions on the variables studied to estimate a VAR model for each of the DAOs.

Table 1: Stationary tests

\begin{tabular}{lll}
\hline Variables & ADF Test & PP Test \\
\hline ret_gas $t$ & $-11.759^{* * *}$ & $-22.866^{* * *}$ \\
DAOstack activity $t$ & $-4.577^{* * *}$ & $-9.225^{* * *}$ \\
DAOHaus activity $t$ & $-2.899^{* *}$ & $-12.031^{* * *}$ \\
\hline
\end{tabular}

${ }^{* * *}$ sig at $1 \%,{ }^{* *} \operatorname{sig}$ at $5 \%$

Table 2: Model results by DAO ecosystem

\begin{tabular}{lllcc}
\hline Model & AIC & BIC & Portmanteau & Granger \\
\hline DAOstack VAR(2) & 36.489 & 36.712 & $90.630^{*}$ & 1.694 \\
DAOHaus VAR(3) & 41.290 & 41.513 & 81.460 & $3.385^{*}$ \\
\hline
\end{tabular}

${ }^{*}$ sig at $10 \%$ level

As we can see in Table 2, for DAOstack we adjusted a VAR(2) and for DAOHaus a VAR(3) model using as criteria AIC, BIC and residuals tests. According to the portmanteau test, we can not reject the hypothesis of white noise for both models at a 5\% significance level, which means that the models account for all the linear information in the data. Regarding to the causality, we find statistical evidence (significant at $10 \%$ ) only for the DAOhaus ecosystem. It means that the effect of the price on the activity is not as strong as to find a strong statistical significance.

Table 3: Forecast error variance decomposition for the activity rate by DAO ecosystem. Proportions of forecast error variance $h$ periods ahead, accounted for by innovations in gas returns and activity rate.

\begin{tabular}{ccccc}
\cline { 2 - 5 }$h$ & \multicolumn{2}{c}{ DAOstack } & \multicolumn{2}{c}{ DAOHaus } \\
\hline$h$ periods & ret_gas $t$ & activity $_{t}$ & ret_gas $_{t}$ & activity \\
\hline 1 & 0.008 & 0.992 & 0.016 & 0.984 \\
5 & 0.033 & 0.967 & 0.034 & 0.966 \\
10 & 0.035 & 0.965 & 0.034 & 0.966 \\
20 & 0.035 & 0.965 & 0.034 & 0.966 \\
\hline
\end{tabular}

Table 3 shows the decomposition of the variance of the forecast error for the activity rate short term $(h=1,5)$, and medium and long term forecasts $(h=10,20)$. The table reveals that the variance of the forecast error in the activity rate is mainly due to own shocks in at least $96.5 \%$, and maximum in $3.5 \%$ to shocks on price returns. In other words, the uncertainty associated with the activity rate forecast of the VAR model comes mainly from its own shocks in both DAO ecosystems. This means that the activity is little sensitive to price movements for both $\mathrm{DAO}$ ecosystems. 

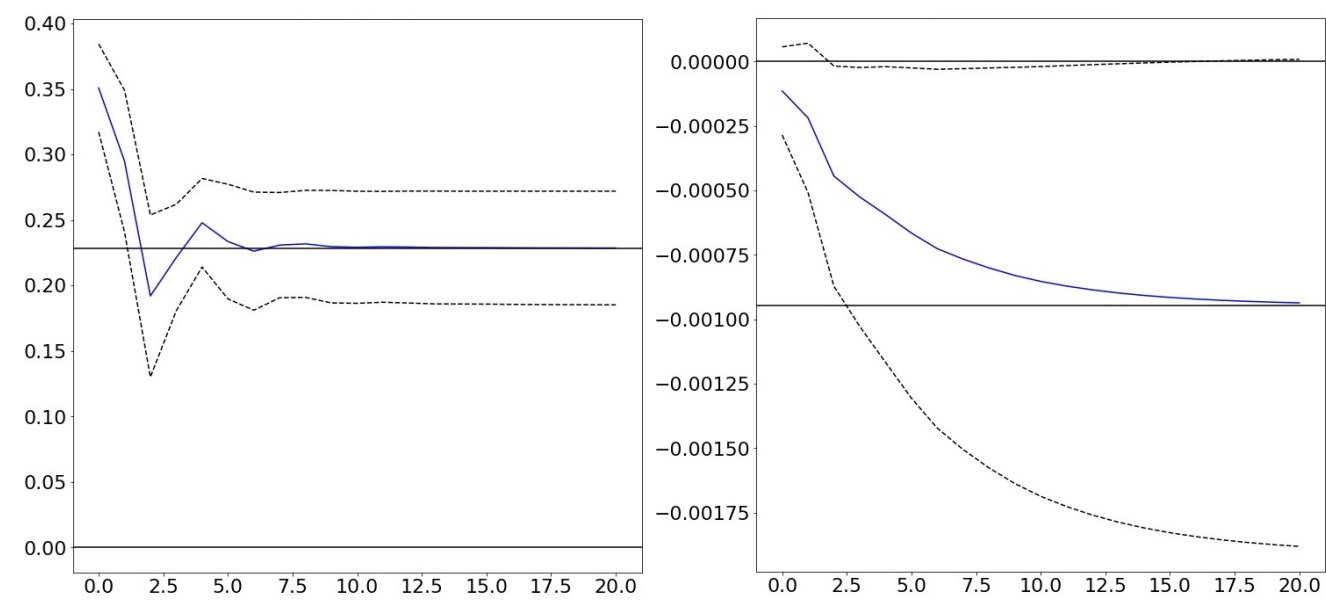

(a) DAOstack impulse-response functions (Left: gas $\rightarrow$ gas, Right: gas $\rightarrow$ activity)
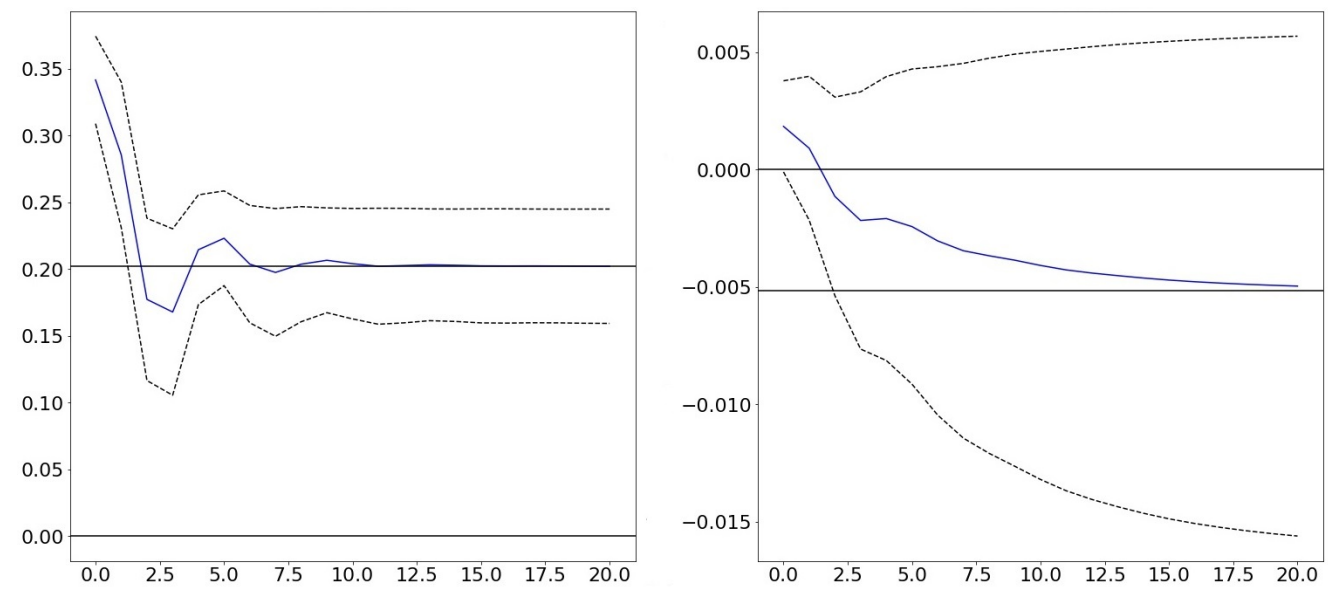

(b) DAOhaus impulse-response functions (Left: gas $\rightarrow$ gas, Right: gas $\rightarrow$ activity)

Figure 2: Accumulated responses of the model variables to an orthogonalized impulse in gas return for the DAO ecosystems.

The impulse response plots in Figures $2 \mathrm{a}$ and $2 \mathrm{~b}$ show that the shapes for both DAO ecosystems are very much alike, which means that the relationships in both cases are quite similar. Then we analyze how the positive shocks in the price returns affect both the price returns and the activity rates. In both, a positive shock (around 0.35, which means a 35\% increase in the daily price) in the gas return leads to increased gas return one period after (around 0.29). Thus, we can expect two consecutive increases in the gas return time series. After that, the impact is not significant, but it leads to a permanent increase in the gas return of around 0.21 in both DAO ecosystems (horizontal line parallel to the $\mathrm{X}$-axis).

Regarding the impulse response functions gas $\rightarrow$ activity in the right-hand side of Figure 2, the shape of both functions is quite similar, even if they are slightly shifted. An important difference is in the responses that are significant in each ecosystem. In the DAOstack system, we observed a significance effect after two days of the shock and even two weeks after. The effect is small, but significant (both intervals determined by the dashed bands are below zero). On the other hand, in DAOhaus, there is no clear significant response according to the $95 \%$ confidence bounds, but we can appreciate that an increase in the gas return causes a simultaneous increase in the activity rate. While it seems counter-intuitive, it makes sense if we consider that some users may hasten to perform their actions because they expect the gas price to keep increasing in the short term (as we saw before).

Interestingly we found both short and mid term effects. This might be due to the combination of both the high-frequency changes in the gas price, that can widely vary even within the day, and the long term decisions that are taken in DAOs, where typically you can vote for a proposal along a few weeks.

Furthermore, we can also observe in both DAO ecosystems that an increase in the gas price causes a decrease in the activity rate that keeps declining as time goes by, although it is not statistically significant. The permanent effect in the activity rate is therefore negative (around -0.0009 in DAOstack and -0.005 in DAOhaus). ${ }^{8}$

\footnotetext{
${ }^{8}$ The mean value of the DAOstack and DAOhaus activity rates were 0.001668 and 0.018197 , respectively, in the period considered.
} 
In general, we found a negative expected sign in the activity due to the shock on price returns (price growth), but not very significant, showing the low sensitivity to price in both DAOs.

\section{DISCUSSION AND CONCLUDING REMARKS}

This work has studied the link between the increase of transaction fees and their impact on user activity within DAOs. Our initial hypothesis established that a surge in fees would negatively affect user activity. This was slight confirmed by the statistical study presented. The statistically significance in data that aggregate different DAOs and users is quite relevant, since within a DAO, there will be users less sensitive to the gas price, while others will be affected and may be pushed away from the system due to this.

Our study focused on DAO activity in order to isolate from those operations directly driven by investment, which would distort the impact on standard users which are not seeking a return of investment from their transaction. Still, of course this may play some role, since some voting activities may have indirect monetary returns for certain DAO users.

The fact that it is a small effect shows that most DAO users need to cope with the surge of fees and absorb the cost individually. This is counter-intuitive in theoretical self-regulated markets, in which raises in the cost of a product (in this case, transactions) typically would reduce its demand until it drops again. The behavior studied is more similar to medicine markets such, where price is inelastic since most consumers will buy the medicine regardless of the price. This is a market signal often understood as the convenience to intervene or regulate the studied market, in order to benefit consumers [3,25]. The mentioned impact is relevant in the development of these platforms particularly in their early years, since it may disincentive DAO use or even trigger abandonment.

Our study has several limitations. For example, we did not account for the impact of the exchange of Ether with fiat currencies, e.g. USD. Moreover, further studies should include other variables that may affect user activity to validate our results, which reveal low sensitivity of activity to the price. Finally, whenever possible, the replication of the experiment with the Aragon platform DAO communities would help to validate our results.

It is worth noting that Ethereum is working to upgrade its technology (in "Ethereum 2.0") to facilitate scalability and reduce the computational effort required to validate and compute transactions [6]. This does not mean fees will disappear, or that the sensitivity to gas price will not continue, but at the very least it is expected it would diminish it. Until then, other solutions have emerged to cope with this situation. For instance, there are smart contracts that tokenize gas, e.g., GST, ${ }^{9}$ storing gas when it is cheap and deploying it when the gas is expensive [21]. Moreover, the use of "sidechains" is becoming more common, i.e. blockchains that run in parallel to a major blockchain (like Ethereum main network) while remaining interoperable with it. A popular one is $\mathrm{xDai},{ }^{10}$ where the gas cost is much lower, and thus facilitates operation. In fact, we can observe a trend of several DAOs and Dapps migrating to use xDai services.

\footnotetext{
${ }^{9}$ https://gastoken.io/

${ }^{10}$ https://www.xdaichain.com/
}

Another example is that Aragon provides a tool for DAOs to vote off-chain and then record the outcome in the blockchain. ${ }^{11}$

Overall, this is a structural problem that may hinder adoption of DAOs and other decentralized services, and thus it should have major consideration in the development of Ethereum 2.0 and other tools in this nascent ecosystem.

\section{ACKNOWLEDGMENTS}

Work supported by the Spanish Ministry of Science, Innovation and Universities [grant: RTI2018-096820-A-100] and by the European Research Council ERC-2017-STG 625 [grant: 759207] through the project P2P Models (https://p2pmodels.eu).

\section{REFERENCES}

[1] 0xNick. 2020. We need to talk about the block gas limit. https://ethereumprice. $\mathrm{org} /$ newsletter/we-need-to-talk-about-the-block-gas-limit/.

[2] Heather M. Anderson. 2007. New introduction to multiple time series analysis. Economic Record 83, 260 (2007), 109-110. https://doi.org/10.1111/j.1475-4932. 2007.00384.x

[3] Kurt R. Brekke, Ingrid Königbauer, and Odd Rune Straume. 2007. Reference pricing of pharmaceuticals. Fournal of Health Economics 26, 3 (2007), 613 - 642. https://doi.org/10.1016/j.jhealeco.2006.11.003

[4] Vitalik Buterin. 2014. A next-generation smart contract and decentralized application platform. https://cryptorating.eu/whitepapers/Ethereum/Ethereum_ white_paper.pdf

[5] Nazli Cila, Gabriele Ferri, Martijn de Waal, Inte Gloerich, and Tara Karpinski. 2020. The Blockchain and the Commons: Dilemmas in the Design of Local Platforms. In Proceedings of the 2020 CHI Conference on Human Factors in Computing Systems (Honolulu, HI, USA) (CHI '20). Association for Computing Machinery, New York, NY, USA, 1-14. https://doi.org/10.1145/3313831.3376660

[6] Mikel Cortes-Goicoechea, Luca Franceschini, and Leonardo Bautista-Gomez. 2020. Resource analysis of Ethereum 2.0 clients. arXiv:2012.14718 [cs.CR] https://arxiv.org/abs/2012.14718

[7] José Eduardo de Azevedo Sousa, Vinícius Oliveira, Júlia Valadares, Glauber Dias Gonçalves, Saulo Moraes Villela, Heder Soares Bernardino, and Alex Borges Vieira. 2020. An analysis of the fees and pending time correlation in Ethereum. International fournal of Network Management n/a, n/a (2020), e2113. https://doi.org/10.1002/nem.2113

[8] David Easley, Maureen O'Hara, and Soumya Basu. 2019. From mining to markets: The evolution of bitcoin transaction fees. Fournal of Financial Economics 134, 1 (2019), 91-109. https://doi.org/10.1016/j.jfineco.2019.03.004

[9] Youssef El Faqir, Javier Arroyo, and Samer Hassan. 2020. An Overview of Decentralized Autonomous Organizations on the Blockchain. In Proceedings of the 16th International Symposium on Open Collaboration. Association for Computing Machinery, New York, NY, USA, Article 11, 8 pages. https://doi.org/10.1145/ 3412569.3412579

[10] Chris Elsden, Bettina Nissen, Karim Jabbar, Reem Talhouk, Caitlin Lustig, Paul Dunphy, Chris Speed, and John Vines. 2018. HCI for Blockchain: Studying, Designing, Critiquing and Envisioning Distributed Ledger Technologies. In Extended Abstracts of the 2018 CHI Conference on Human Factors in Computing Systems (CHI EA '18). Association for Computing Machinery, New York, NY, USA, 1-8. https://doi.org/10.1145/3170427.3170602

[11] Youssef Faqir-Rhazoui, Javier Arroyo, and Samer Hassan. 2021. A scalable voting system: Validation of holographic consensus in DAOstack. In Proceedings of the Hawaii International Conference on System Sciences, Vol. 54. University of Hawaii, Manoa, Hawaii, 5557. https://doi.org/10.24251/HICSS.2021.676

[12] Primavera De Filippi and Samer Hassan. 2016. Blockchain technology as a regulatory technology: From code is law to law is code. First Monday 21, 12 (Nov. 2016), 23 pages. https://doi.org/10.5210/fm.v21i12.7113

[13] Primavera De Filippi and Aaron Wright. 2018. Blockchain and the law: The rule of code. Harvard University Press, Cambridge, Massachusetts; London, England. https://doi.org/10.2307/j.ctv2867sp

[14] C. W. J. Granger. 1969. Investigating causal relations by econometric models and cross-spectral methods. Econometrica 37, 3 (1969), 424-438. https://doi.org/10. $2307 / 1912791$

[15] Samer Hassan and Primavera De Filippi. 2021. Decentralized autonomous organizations (Glossary of distributed technologies). https://policyreview.info/openabstracts/decentralised-autonomous-organisation

[16] Sarah Kordyban. 2020. Why Ethereum's gas prices have skyrocketed. https: //hedgetrade.com/ethereums-gas-prices-skyrocket/.

${ }^{11}$ The tool is called Snapshot https://snapshot.page 
[17] Caitlin Lustig. 2019. Intersecting imaginaries: visions of decentralized autonomous systems. Proceedings of the ACM Human-Computer Interaction 3, CSCW, Article 210 (Nov. 2019), 27 pages. https://doi.org/10.1145/3359312

[18] James G. MacKinnon. 1994. Approximate asymptotic distribution functions for unit-root and cointegration tests. Fournal of Business \& Economic Statistics 12, 2 (1994), 167-176. https://doi.org/10.1080/07350015.1994.10510005

[19] James G. MacKinnon. 2010. Critical values for cointegration tests. Working Paper 1227. Economics Department, Queen's University. https://ideas.repec.org/p/qed/ wpaper/1227.html

[20] Malte Möser and Rainer Böhme. 2015. Trends, tips, tolls: A longitudinal study of Bitcoin transaction fees. In Financial Cryptography and Data Security, Michael Brenner, Nicolas Christin, Benjamin Johnson, and Kurt Rohloff (Eds.). Springer Berlin Heidelberg, Berlin, Heidelberg, 19-33.

[21] Matthias Nadler. 2020. A quantitative analysis of the Ethereum fee market: How storing gas can result in more predictable prices. https://wwz.unibas.ch/fileadmin/user_upload/wwz/00_Professuren/Schaer DLTFintech/Lehre/Teaching_Page/Nadler_MasterThesis_2020-1_copy.pdf

[22] Satoshi Nakamoto. 2009. Bitcoin: A peer-to-peer electronic cash system. http: //www.bitcoin.org/bitcoin.pdf

[23] G. A. Pierro and H. Rocha. 2019. The influence factors on ethereum transaction fees. In 2019 IEEE/ACM 2nd International Workshop on Emerging Trends in Software Engineering for Blockchain (WETSEB). IEEE, Montreal, QC, Canada, 24-31. https: //doi.org/10.1109/WETSEB.2019.00010

[24] Michal Polasik, Anna Iwona Piotrowska, Tomasz Piotr Wisniewski, Radoslaw Kotkowski, and Geoffrey Lightfoot. 2015. Price fluctuations and the use of Bitcoin
An empirical inquiry. International fournal of Electronic Commerce 20, 1 (2015), 9-49. https://doi.org/10.1080/10864415.2016.1061413

[25] Franco Sassi, Annalisa Belloni, Andrew J Mirelman, Marc Suhrcke, Alastair Thomas, Nisreen Salti, Sukumar Vellakkal, Chonlathan Visaruthvong, Barry M Popkin, and Rachel Nugent. 2018. Equity impacts of price policies to promote healthy behaviours. The Lancet 391, 10134 (2018), 2059 - 2070. https://doi.org/ 10.1016/S0140-6736(18)30531-2

[26] Christopher A. Sims. 1980. Macroeconomics and reality. Econometrica 48, 1 (1980), 1-48. https://doi.org/10.2307/1912017

[27] Skipper Seabold and Josef Perktold. 2010. Statsmodels: Econometric and Statistical Modeling with Python. In Proceedings of the 9th Python in Science Conference, Stéfan van der Walt and Jarrod Millman (Eds.). Skipper Seabold, Online, $92-96$. https://doi.org/10.25080/Majora-92bf1922-011

[28] Sarah Underwood. 2016. Blockchain beyond Bitcoin. Commun. ACM 59, 11 (2016), 15-17. https://doi.org/10.1145/2994581

[29] Alex Van de Sande. 2020. Out of gas: We're shutting down uniLogin. https://medium.com/universal-ethereum/out-of-gas-were-shutting-downunilogin-3b544838df1a.

[30] Gavin Wood et al. 2014. Ethereum: A secure decentralised generalised transaction ledger. https://cryptopapers.info/assets/pdf/eth_yellow_byzantium.pdf. Ethereum Project Yellow Paper 151, 2014 (2014), 1-32.

[31] Working Group Ethereum. 2014. A secure decentralised generalised transaction ledger. , 32 pages. http://gavwood.com/Paper.pdf 